\title{
Accuracy of MRI (TLICS vs AOSIS) in assessment of thoracolumbar spine injuries for guiding treatment
}

\author{
Mona Ali Mohamed Ali Nagi* (i) and Mai Mohamed Sedik Sakr
}

\begin{abstract}
Background: The thoracic segment represents the most common area fractured in the whole spine. Complete neurological deficits are commonly associated with thoracic injuries possibly due to a relatively small canal diameter as compared to the cervical or lumbar spine. Magnetic resonance is the gold standard of imaging, especially in patients suffering from neurological deficits as well as in soft tissue assessment mainly the disc, ligaments, and neural elements. The thoracolumbar injury classification and severity score system (TLICS) and the thoracolumbar AO Spine injury score (TLAOSIS), are two scoring systems designed to help surgeons in management plans of thoraco-lumbar injuries. The aim of our study is to compare these two main thoracolumbar injury classification systems in deciding the management strategies in thoraco-lumbar injuries. This study is a retrospective study that included 70 patients (42 males and 28 females) who suffered acute traumatic vertebral fractures. All patients underwent MRI including T1WI, T2W and STIR sequences. The MRI was viewed by two independent radiologists of 5- and 10-years' experience and compared to surgical decisions.

Results: Out of 70 patients included in our study, the TL AOSIS matched treatment recommendation in 62 patients (88.6\%), and the TLICS matched in 60 patients (85.7\%). The TL AOSIS achieved sensitivity 95\%, specificity 80\%, while the TLICS achieved sensitivity $72.2 \%$, specificity $100 \%$.

Conclusion: Both TL AOSIS and TLICS have very close results in their reliability for guiding treatment strategy, yet TL AOSIS matched treatment recommendation more than TLICS, with sensitivity more than TLICS, while TLICS had more specificity.
\end{abstract}

Keywords: Thoracolumbar trauma, Thoracolumbar AO Spine injury score, Thoracolumbar injury classification, Severity score system

\section{Introduction}

Acute spine trauma has a devastating effect on both patients as well as their families [1].

Thoracolumbar (TL) fractures are the commonest traumatic injuries of the spinal column, usually involving the levels T10 to L2 [2]. This is possibly attributed to the small canal diameter contrary to the cervical or lumbar spine, which have a relatively wider canal [3].

\footnotetext{
*Correspondence: mona.babydoc@yahoo.com

Radio-Diagnosis Department, Faculty of Medicine, Ain Shams University, 38 Ramsis Street, Abassia, Cairo, Egypt
}

Conventional radiographs and computed tomography (CT) are the initial imaging modalities used in the diagnosis of spinal injuries [4].

Because MRI can assess nerve root, spinal cord, and posterior cruciate ligament (PLC) damage, it plays an important role after initial evaluation with $\mathrm{CT}$ and/or $\mathrm{X}$-rays, especially when neurological damage is suspected [5].

The TLICS system was developed to aid in the diagnosis and decision-making of thoraco-lumbar spinal trauma [6]. This allows a more accurate diagnosis and, consequentially, better management of the case [7]. 
TLICS has three main contributors: injury morphology, status of the posterior ligamentous complex (PLC), and neurological status (Table 1) [8].

The three characteristics of TLICS are evaluated in isolation, and after summation, a final injury description and injury severity score are obtained, guiding treatment. A score of three or less suggests conservative management of the patient. A score of five or more points suggests that surgery is the best option for treatment. Patients with a score of four points can be treated either conservatively or surgically depending on several factors, including the

Table 1 Shows TLCS classification [8]

\begin{tabular}{ll}
\hline Injury category & Point value \\
\hline Injury morphology & 1 \\
Compression & 2 \\
Burst & 3 \\
Translation or rotation & 4 \\
Distraction & \\
PLC status & 0 \\
Intact & 2 \\
Suspected & 3 \\
Injured & \\
Neurological status & 2 \\
Nerve root involvement & 3 \\
Spinal cord or conus medullaris injury & 3 \\
Incomplete & 2 \\
Complete & 3 \\
Cauda equina syndrome & \\
\hline
\end{tabular}

surgeon's preference, additional injuries, patient age, and the presence of any pre-existing spinal disease [9].

The AO thoracolumbar classification system was formulated by Magerl et al. [10] then updated by Vaccaro et al. [11]. After a while, Kepler et al. [4] and Vaccaro et al. [12] formulated a new surgical algorithm and published the thoracolumbar AO spine Injury score (TL AOSIS) (Tables 2, 3).

In this study, we evaluated the usefulness of TLICS in surgical decisions with and without inclusion of neurological status, thus emphasizing the importance of radiological investigations as well as comparing the sensitivity and specificity of the TLICS scoring system to the AOSIS.

\section{Methods}

\section{Study design and population}

Our study included 70 patients older than 18 years suffering from acute thoracic and/or lumbar vertebral injuries.

The exclusion criteria included general contraindications for MRI, e.g. claustrophobia, an implanted magnetic device or pacemakers. Pathological fractures (tumors, infection, etc.) without trauma as well as any surgical intervention before MRI.

\section{Preparation and protocol}

All patients were subjected to:

- Full history before MRI was done.

- Detailed clarification of imaging procedure.

- The patient was positioned supine on the MRI table.

Table 2 The AO Spine thoracolumbar spine injury classification system. [4]

\begin{tabular}{ll}
\hline Type A (compression fractures) & \\
A0 & Minimal injuries such as transverse process fractures \\
A1 & Wedge compression \\
A2 & Pincer compression injury \\
A3 & Incomplete burst fracture: fracture that only involves a single endplate \\
A4 & Complete burst fracture: fracture that involves both endplates \\
Type B (band injuries) & \\
B1 & Osseous disruption of the tension band \\
B2 & Posterior tension band injury including ligamentous \\
B3 & Anterior tension band injury \\
Type C (displacement or dislocation) & \\
N (neurological status) & \\
N0 & Neurologically intact \\
N1 & Transient neurological deficit \\
N2 & Radicular symptoms \\
N3 & Incomplete spinal cord injury or any degree of cauda equina \\
N4 & Complete spinal cord injury \\
Nx & Neurological status is unknown \\
\hline
\end{tabular}


Table 3 The thoracolumbar AO Spine injury score (TL AOSIS) [4]

\begin{tabular}{ll}
\hline Subgroup & Points \\
\hline Type A (compression injuries) & \\
A0 & 0 \\
A1 & 1 \\
A2 & 2 \\
A3 & 3 \\
A4 & 5 \\
Type B (tension band injuries) & \\
B1 & 5 \\
B2 & 6 \\
B3 & 7 \\
Type C (Translational fracture) & \\
Neurological status & 8 \\
N0 & \\
N1 & \\
N2 & 0 \\
N3 & 1 \\
N4 & 2 \\
Nx & \\
M (patient-specific modifiers & \\
M1 & \\
M2 & 4 \\
\hline
\end{tabular}

- MRI technique: MRI study was performed by using a $1.5 \mathrm{~T}$ machine (Achieva, Philips medical system, Eindhoven, Netherlands).All patients underwent the MRI examination within the first week after trauma. The study took 15-20 min. The protocol for spinal injury including sagittal T1 weighted (T1W), T2 weighted (T2W) spin echo sequences, sagittal short tau inversion recovery (STIR) sequence and axial T2W sequences.

- Image analysis:

T1W images: Offer an excellent anatomic overview and better characterization of osseous fractures.

T2WI sequence and STIR images are sensitive for edema detection, soft tissue and ligamentous injuries. After MRI have been done images were viewed by two independent radiologists of 5 and 10 years' experience.

- Statistical Analysis: Data were collected, revised, coded and entered to the Statistical Package for Social Science (IBM SPSS) version 23. The quantitative data were presented as mean, standard deviations and ranges when their distribution found parametric. Also, qualitative variables were presented as number and percentages. The comparison between groups regarding qualitative data was done by using
Chi-square test. The comparison between two independent groups with quantitative data and parametric distribution were done by using Independent $t$ test while the comparison between two independent groups with quantitative data and non-parametric distribution were done by using Mann-Whitney.

Receiver operating characteristic curve (ROC) was used to assess the sensitivity, specificity, positive predictive value (PPV), negative predictive value (NPV) and area under curve (AUC) of TLICS score between the two groups.

The confidence interval was set to $95 \%$ and the margin of error accepted was set to $5 \%$. So, the $p$ value was considered significant as the following:

$P>0.05$ : Non significant, $P<0.05$ : Significant $<0.01$ : Highly significant.

\section{Results}

Our study included a total of 70 patients, 28 were females $(40 \%)$ and 42 males (60\%) with age ranging from 18 to 78 years and mean $41.20 \pm 17.50$.

The relation between mode of injury and injury morphology was detected, showing that compression fracture (Fig. 1) was caused mainly by fall on back while burst fracture caused mainly by fall from height, translation fracture mainly by vehicle accidents (Fig. 2)while distraction injury caused mainly by vigorous over flexion (Fig. 3). In our study 12 patients out of 70 (17.1\%) had vertebral trauma at T1 to T10 level, 42 patients out of 70 (60\%) had vertebral trauma at T11 to L2 level, 16 patients out of $70(22.9 \%)$ had vertebral trauma at L3 to L5 level (Fig. 1).

Neurological status of patients was assessed showing that 10 out of 70 patients were neurologically free, 42 out of 70 patients had root injury symptoms or signs, 6 of them had incomplete cord injury (Fig. 2) while 10 had conus injury and 2 had complete cord injury.

The TLICS score ranged from 1 to 7 with median two, it was 4 in 24 patients (34.3\%). Ten of our patients were neurologically free; all of them received $<4$ points on TLICS score. In patients with neurological symptoms, 34 of 60 received $<4$ points, 24 received $>4$ points and 2 received 4 points.

While in TL AOSIS system, the score was less than 4 in 24 patients (34.3\%), (4-5) in 14 patients $(20 \%)$ and $>5$ in 32 patients (45.7\%). All neurologically free patients (10 out of 70) received $<4$ points. Among the patients who suffered from neurological symptoms (60 of 70 patients), 32 of 60 received $>5$ points, 14 received 4 or 5 points, 14 received $<4$ points. $P$ value $=0.000$ in both TLICS and TL AOSIS systems. 


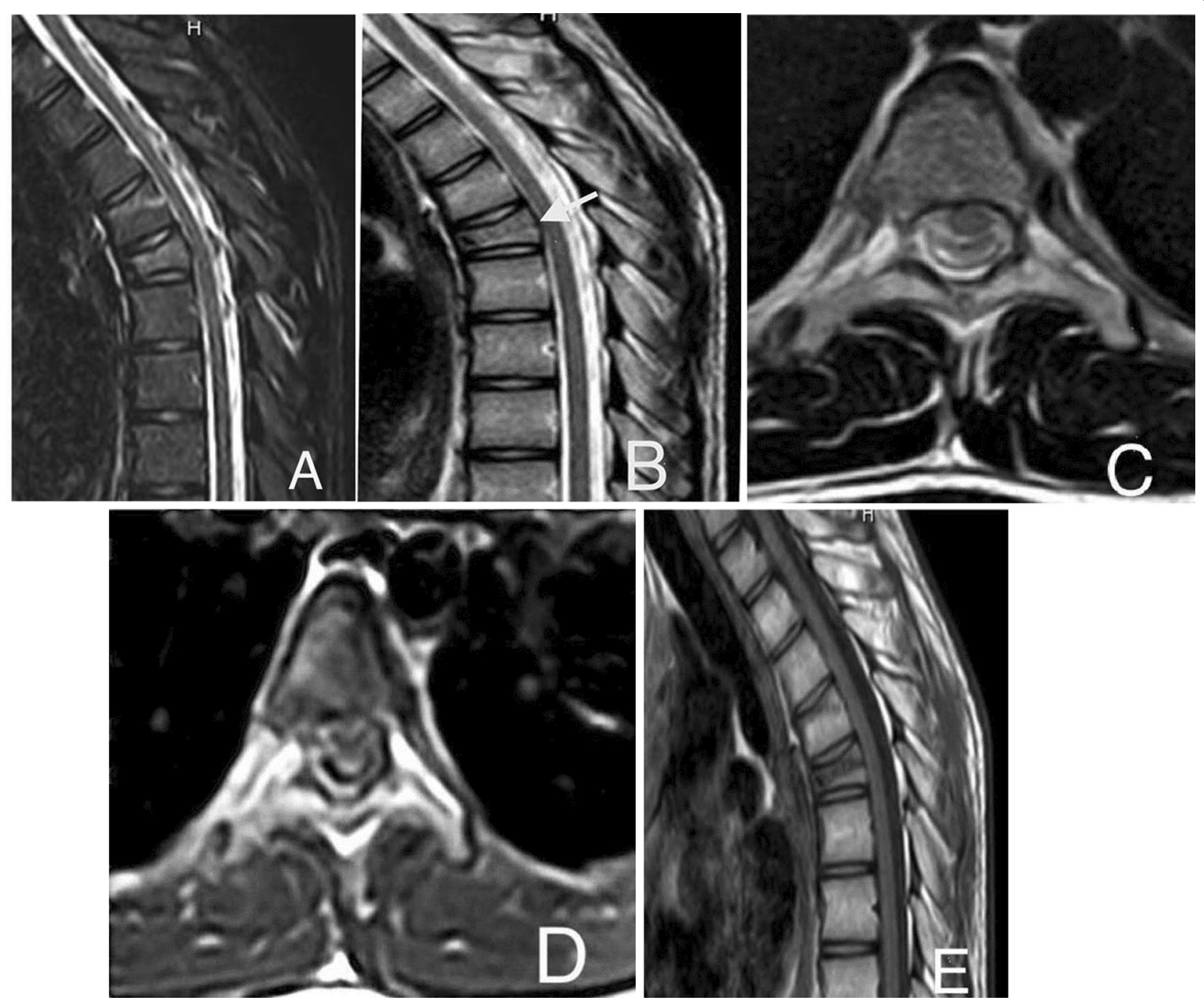

Fig. 1 18-year-old male patient presented after fall from height with T6 compression fracture. a Sagittal STIR image shows hyper intense bone marrow signal at T6 vertebral body indicating edema. b Sagittal T2WI shows decrease height of T2 vertebra with intact posterior cortex indicating compression fracture (white arrow). c Axial T2WI of T6 vertebra. d Axial T1WI of T6 vertebra. e Sagittal T1WI of T6 vertebra. His TLICS score was 1, TL $\mathrm{AOSIS}=\mathrm{A} 1 \mathrm{NO}$ was 1 also and he was treated conservatively

In our study 36 patients out of 70 underwent surgical treatment (S group) while 34 received conservative treatment (C group) (Table 5). For TL AOSIS system application patients were divided into 3 groups $(A, B, C)$ as well as sub groups for detailed morphological description of injury. Also the neurological status is divided into 5 groups (N0, N1, N2, N3, N4) (Table 3). TL AOSIS score in (C group) ranged from 1 to 9 with median 3 , while in (S group) ranges from 3 to 12 with median 8 . The TLICS matched treatment recommendation in 60 patients (85.7\%), while the TL AOSIS matched treatment recommendation in 62 patients $(88.6 \%)$ with high significant value (Table 4).

Comparison between TLICS score without inclusion of the neurological status i.e. injury morphology and PLC status solely and TLICS score with the inclusion of neurological status revealed that the former gives a highly significant relation with correct decision making dividing the patients into two group ( $S$ group) that underwent surgical operation with median 5 and IQR (2-6), and (C group) that were treated conservatively with median 2 and IQR (Table 5). 

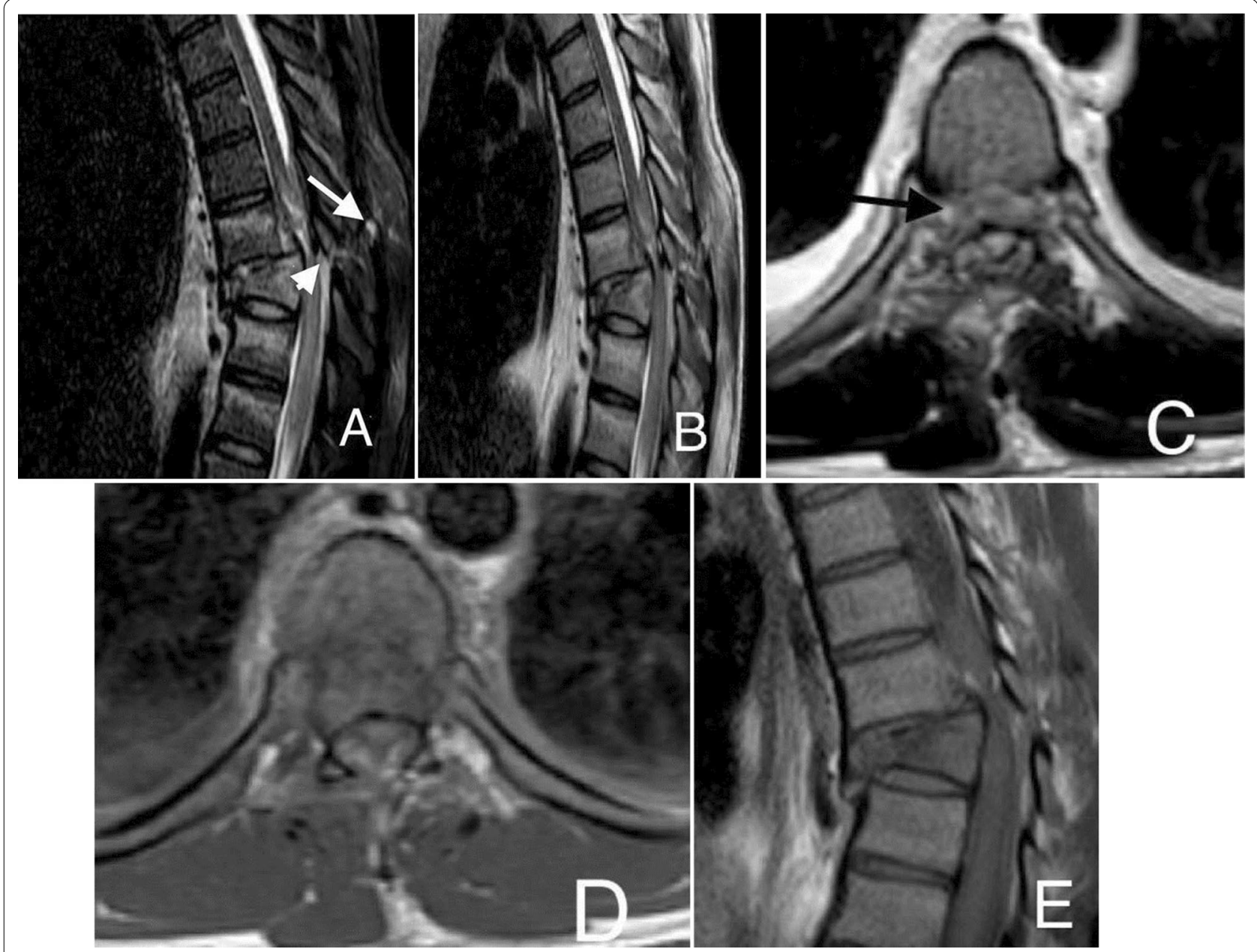

Fig. 2 A 30 year-old male presented after motor accident with T7 anterior translation over T8 with ligamentous injury (PCL injury) white arrow with consequent severe cord compression by extradural hematoma (incomplete cord). a Sagittal STIR shows ligamentum flavum (white arrowhead), supra-spinous ligamentous injury (white arrow), cord compression with high signal intensity. b Sagittal T2WI and c axial T2WI shows extradural hematoma (black arrow) compressing spinal cord, compromising both nerve roots. d Axial T1WI of T7 vertebra. e Sagittal T1WI. His TLICS score was nine, $\mathrm{TL} A O S I S=C N 3$, score was 12 and underwent surgical intervention

All neurologically free patients $(22.7 \% n=10)$ had TLICS $<4.18 .2 \%$ of patients $(n=8)$ suffering from conus medullaris injury had TLICS $<4$ while in $7.7 \%$ $(n=2)$ had TLICS $>4$, patients. In patients suffering from root injury $(59.1 \% n=26)$ had TLICS $<4$ and $(61.5 n=16)$ had TLICS $>4$. Patients with incomplete $(23.1 \% n=6)$ and complete cord injury $(7.7 \% n=2)$ all had TLICS $>4$.

Based on the area under ROC curve of 0.879 the estimated TLICS score cutoff point is shown to be $>3$ very near to 4 which divides TLICS score into two groups $\mathrm{S}$ and $\mathrm{C}$ group Hence, TLICS score achieved sensitivity $=77.2 \%$, specificity $=100 \%$, positive predictive value $=100$, and negative predictive value $=77.3$ (Fig. 4a).

On the other hand the area under ROC curve of 0.923, TL AOSIS classification cut off point was $>4$, achieving sensitivity $=95 \%$, specificity $=80 \%$, positive predictive value $=86.4$ and negative predictive value $=92.3$ (Fig. 4b). 

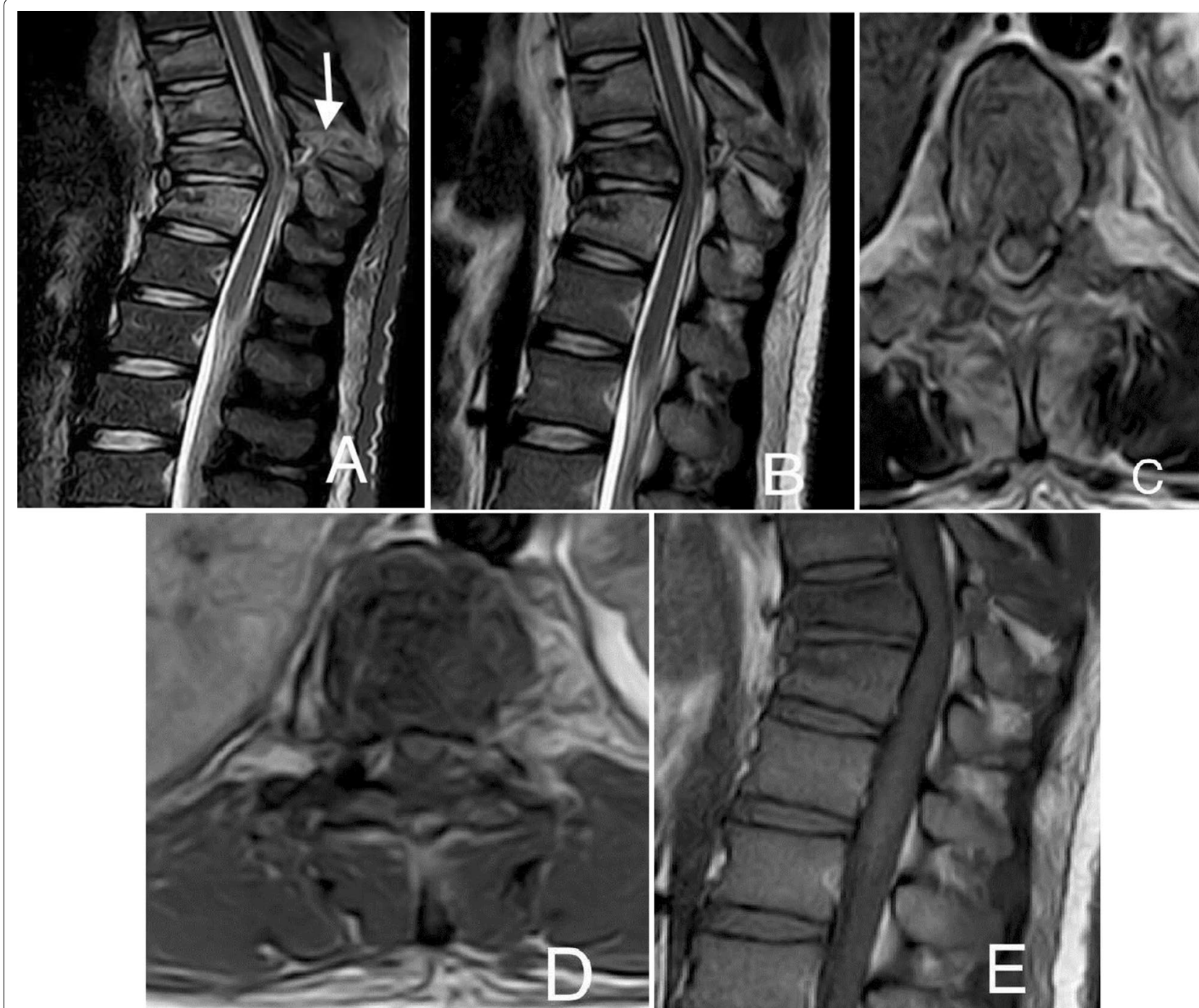

Fig. 3 A 28-year-old male patient presented after fall from height T9/T10 widened interspinous space with distraction injury and PLC injury with cord compression. a Sagittal STIR shows complete posterior ligamentous disruption (interspinous, supraspinous, ligamentum flavum), increase inter-spinous space (white arrow), cord compression with high signal intensity. b Sagittal T2WI, c: Axial T2WI, d Axial T1WI, e Sagittal T1WI of T10 vertebra. TLICS score was 10, TL AOSIS=B1 N3, score was 9 and treated surgically

Table 4 The comparison of the matching treatment recommendation of both TL AOSIS and TLICS systems

\begin{tabular}{|c|c|c|c|c|c|c|c|c|c|}
\hline & \multicolumn{3}{|l|}{ Overall } & \multicolumn{3}{|c|}{ Neurologically intact } & \multicolumn{3}{|c|}{ Neurologic deficit } \\
\hline & Match & Mismatch & $\%$ & Match & Mismatch & $\%$ & Match & Mismatch & $\%$ \\
\hline TLICS & 60 & 10 & 85.7 & 10 & 0 & 100 & 26 & 34 & 50 \\
\hline TL AOSIS & 62 & 8 & 88.6 & 10 & 0 & 100 & 46 & 14 & 100 \\
\hline$\times 2$ & 0.255 & & & - & & & 13.889 & & \\
\hline$P$ value & 0.614 (NS) & & & - & & & $<0.001(H S)$ & & \\
\hline
\end{tabular}


Table 5 Relation between TLICS score and decision making even without inclusion of neurological status

\begin{tabular}{|c|c|c|c|c|c|}
\hline & $\begin{array}{l}\text { S group } \\
\text { No. }=36\end{array}$ & $\begin{array}{l}\text { C group } \\
\text { No. }=34\end{array}$ & Test value & $P$ value & Sig \\
\hline \multicolumn{6}{|c|}{ TLICS score without neurological status } \\
\hline $\begin{array}{l}\text { Median (IQR) } \\
\text { Range }\end{array}$ & $\begin{array}{l}5(2-6) \\
1-7\end{array}$ & $\begin{array}{l}2(1-2) \\
1-3\end{array}$ & $-5.582 \neq$ & 0.000 & HS \\
\hline TLICS $<4$ & $10(27.8 \%)$ & $34(100.0 \%)$ & $39.066^{*}$ & 0.000 & HS \\
\hline TLICS $>4$ & $24(66.7 \%)$ & $0(0.0 \%)$ & & & \\
\hline 4 & $2(5.6 \%)$ & $0(0.0 \%)$ & & & \\
\hline \multicolumn{6}{|c|}{ TLICS score with neurological status } \\
\hline Median (IQR) & $7(5-9)$ & $3(3-4)$ & $-6.255 \neq$ & 0.000 & $\mathrm{HS}$ \\
\hline Range & $4-10$ & $1-5$ & & & \\
\hline
\end{tabular}

$P$ value $>0.05$ : Non significant; $P$ value $<0.05$ : Significant; $P$ value $<0.01$ : Highly significant

${ }^{*}$ Chi-square test; $\neq$ : Mann-Whitney test

\section{Discussion}

According to our research, the TLICS scoring system was in keeping with the clinical decision in all the patients who were treated conservatively. All 17 patients who were treated conservatively had had a TLICS score more than four. Only five of 18 patients (27.8\%) underwent surgical intervention despite having a TLICS score $>4$, this could be attributed to their neurological status, which was not included so TLICS score matched surgical treatment in $66.7 \%$ of patients. After inclusion of neurological status in TLICS score it accurately matched surgical treatment in $100 \%$ of patients, their TLICS score after neurological status inclusion $\geq 4$. This was an advantage in our study to evaluate the high significance of utilizing injury morphology and PLC injury solely in decision-making. Thus using the TLICS scoring achieved sensitivity $77.2 \%$, specificity $100 \%$, positive predictive value 100 , and negative predictive value 77.3 .

This suggests that use of the TLICS may identify unstable injuries that would otherwise be missed and directs these situations towards surgical care. As a corollary, it suggests that the conservative treatment of unstable injuries (TLICS > 4) will likely fail due to kyphosis, deformity, and pain.

Our study matched with Pizones et al. [13] in a study included fifty-eight vertebral fractures (38 surgical, 20 conservative), of which $50 \%$ were males with average age of 40.4 years. He concluded that MR imaging accuracy in the diagnosis of traumatic PLC injuries had a total sensitivity and specificity of $91 \%$ and $100 \%$ respectively, with $100 \%$ accuracy in diagnosis of surgical fractures. Thus, MRI is a very useful tool in the evaluation of acute thoracolumbar fractures, as it allows a better visualization of

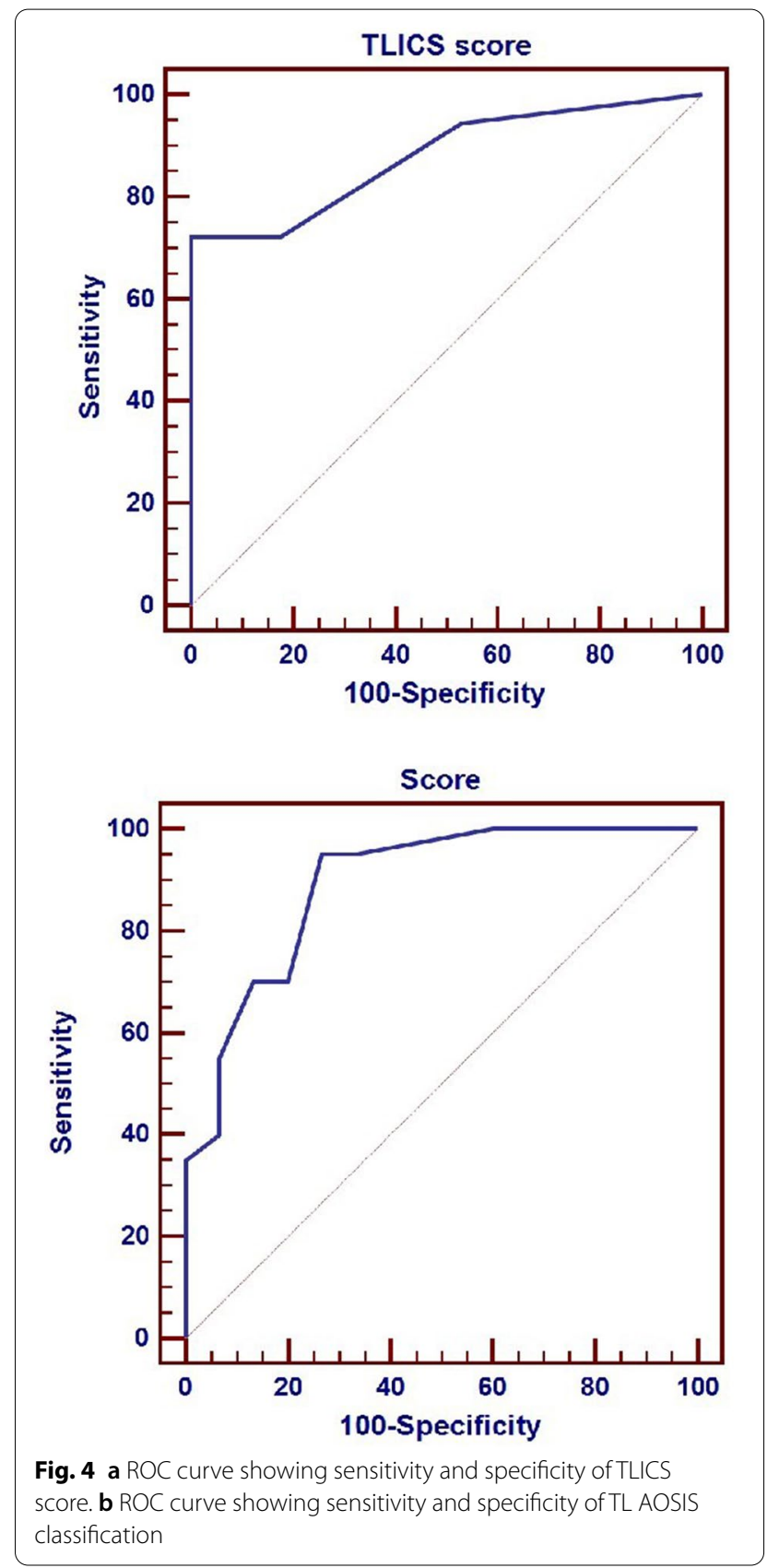

the posterior complex integrity presenting extra data in comparison to the other conventional diagnostic tools. Pizones et al. [13] differ from our study that his study was a prospective study, data was analyzed before and after MRI examination, focusing on: diagnostic changes in classification, occult injuries and differences in treatment decision.

Our results also match with Wood et al. [2] in a study of 47 patients, demonstrated that surgical treatment of stable burst fractures (TLICS $<4$ ) did not improve clinical 
outcomes and increased the risk of complications for the patient.

In a retrospective study done by Pneumaticos et al. [14] enrolling 58 patients with TL fractures (group A and B) treated conservatively and evaluated over a follow-up period of 28 months. He concluded that conservative treatment of cases with a TLICS score of 4 can be safely applied and is equally as valid to those scoring $<3$.Thus, his results are in keeping with ours that TILCS scoring is effective in decision making in TL spine fractures without neurological deficits.

Yuksel et al. [15] in a retrospective analysis of 55 patients with TL burst fractures aimed to evaluate the reliability of recommendations in the surgical management of unstable TL burst fractures using both the TLICS and the AO System.He stated that all patients suffering from neurological deficits (18 patients) received a TLICS $>4$. Patients with incomplete spinal cord injury (14) all received a TLICS score $>4$, but according to AO system, eight of them received 4 points. None of the neurologically free patients (37) received $<4$ points of TLICS yet 18 of them received 3 AO points, to whom AO recommends conservative treatment despite the fact that they had unstable burst fractures. He concluded that the TLICS recommendations are more reliable than those of AO especially in aiding the surgical decisions regarding the unstable thoracolumbar burst fractures without neurological deficit. These results are not matching our study that revealed that both TL AOSIS and TLICS are very close as regards their reliability for guiding treatment plans, yet TL AOSIS matched treatment decisions more than TLICS.

In another retrospective study by Dodwad et al. [16] that included 201 patients with thoracolumbar junction injuries revealed that the TLICS system agreed with the treatment plan at their institute in $98 \%$ of the time in conservatively managed patients and in $78 \%$ of the time in patients who underwent surgical intervention. This is in keeping with a study by An et al. [17] whose results revealed revealing that TLICS matched $87.27 \%$ patients without neurological deficit. This is also matching with our results as the TLICS matched treatment recommendation in 60 patients $(85.7 \%)$.

Joaquim et al. [7] studied 49 patients prospectively treated surgically for thoracic and lumbar spine trauma The TLICS score range from 2 to 9 (average of 6.2). Fortyseven of $49(96 \%)$ patients had a TLICS score greater than 4, suggesting surgical treatment, the TLICS score treatment recommendation matched surgical treatment in 47 of 49 patients $(96 \%)$, the TLICS score $(P<0.0001)$, his values were comparable to our study could be attributed to near number of patients.
Our study showed that the two classification systems have an agreeable output in treatment recommendations and decision making for most patients with thoracolumbar spine injuries. It should be noted that in the group without neurological deficit all of patients received $<4$ of TL AOSIS and also in TLICS score, all of them treated conservatively which was recommended by TLICS and TL AOSIS classification.

In our study, the TLICS matched the management plan in 60 patients $(85.7 \%)$ while the TL AOSIS matched treatment recommendation in 62 patients (88.6\%). TLICS score showed sensitivity $77.2 \%$ compared to $95 \%$ in TL AOSIS, specificity $=100 \%$ compared to $80 \%$, positive predictive value $=100$ compared to 86.4 in TL AOSIS, and negative predictive value $=77.3 \%$ compared to $92.3 \%$ in TL AOSIS. Our study matched with Pizones et al. [13] who reported that MR imaging efficiency in diagnosis of traumatic PLC injuries has achieved overall sensitivity and specificity of $91 \%$ and $100 \%$ respectively, with $100 \%$ accuracy in diagnosis of surgical fractures.

Our study is also in keeping with Joaquim et al. [6] that included 214 patients, 148 of them were treated conservatively $(\mathrm{C})$ and 66 were surgically (S) treatedThe TLICS matched the management recommendation in $97.9 \%$ of patients who were treated conservatively. In another study by Joaquim et al. [7], the TLICS score treatment decision matched surgical treatment in 47 of 49 patients (96\%).

If surgery was the choice for these cases it would due to the treating surgeon's concerns for fracture comminution and the probability of progressive deformity, Some our cases had burst fracture with TLICS not matching the treatment plan while TL AOSIS matched (Figs. 5, 6). Those cases were treated surgically to decrease the probability for kyphosis deformity compared with conservative treatment. The TL AOSIS system differentiate between the rather benign incomplete burst fractures involving one end plate (typically treated conservatively), and the rather unstable complete burst fracture [18]

Also in a study done by An et al. [17] 110 patients were studied. The TL AOSIS matched the recommendations for the management of 108 patients (98.18\%) while TLICS matched in 96 patients (87.27\%). In patients who were neurologically free, according to the TL AOSIS system, 12 of 62 received more than 5 points, 12 received 4 or 5 points, and 38 received less than 4 points. On the contrarily, according to the TLICS system, 12 of 62 received five or more points, 50 received $\leq 3$ points. The TL AOSIS matched the management plan of 60 patients (96.77\%), while the TLICS matched management recommendation in 48 patients (77.42\%). For the patients with neurological deficits when TL AOSIS system was applied, 


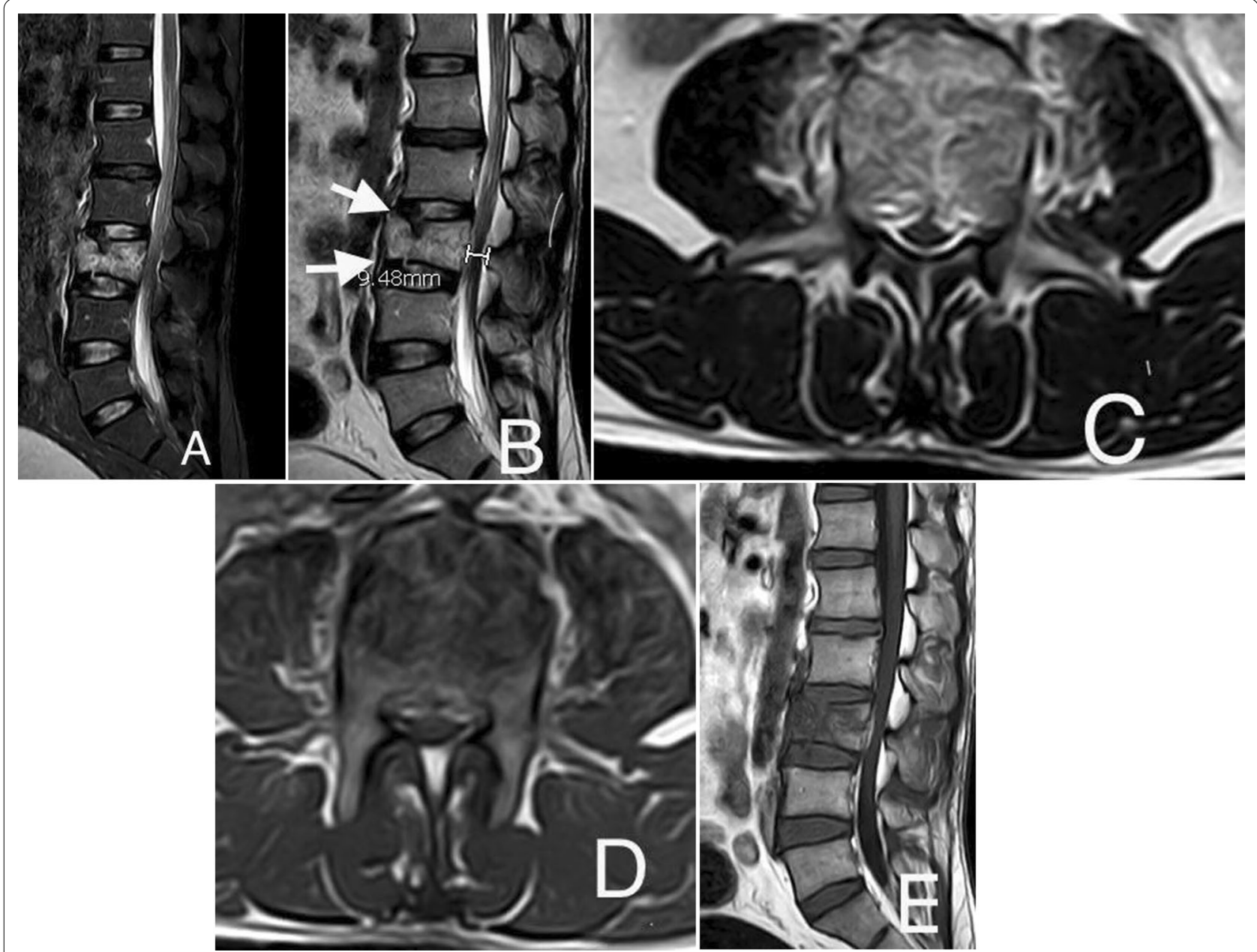

Fig. 5 A 43-year-old male patient presented after fall from height. a Sagittal STIR shows L3 burst with intact PLC. b Sagittal T2WI shows fracture of both superior and inferior end plates (white arrows) yet without involvement of posterior wall of vertebral body. c Axial T2WI. d Axial T1WI. e Sagittal T1WI, TLICS score was two, TL AOSIS = A2 N3 (split/pincer fracture), Secondary canal stenosis is noted with the spinal canal is measuring about $9.48 \mathrm{~mm}$. The score was six and treated surgically so TLICS didn't match treatment while TL AOSIS matched the treatment

36 of 48 received more than 5 points, and 12 received 4 or 5 points. While regarding the TLICS system, 22 received more than 4 points, and 26 received 4 points.

The neurological preservation is a vital goal in the acute management of patients, collectively; these records support the preliminary conservative management of stable burst fractures with the use of an external orthosis. The criteria for conversion to surgical treatment remain unclear, especially when we considered axial back pain as a potential cause of failure in the conservative treatment. Chronic pain is a complex subject in spinal trauma, not accessed in our study, which requires long-term follow-up and functional status questionnaires evaluation. Prospective application of the TLICS system, with consistently defined injuries, will better elucidate the potential differences between surgical and conservative treatment.

Our study has few limitations including by the relatively small number of patients as well as its retrospective nature and the limited clinical follow-up. We recommend more studies to be done in a prospective manner are important to assess the prognostic value of the classification scoring systems on patient outcomes.

However, it was difficult to prove the validity of the clinical consequences prospectively according to the TLICS classification because patients with high TLICS score that required surgical intervention could not be observed without surgery. Multi-institute studies are needed because of possibility of biases for treatment decisions within a single institute. 

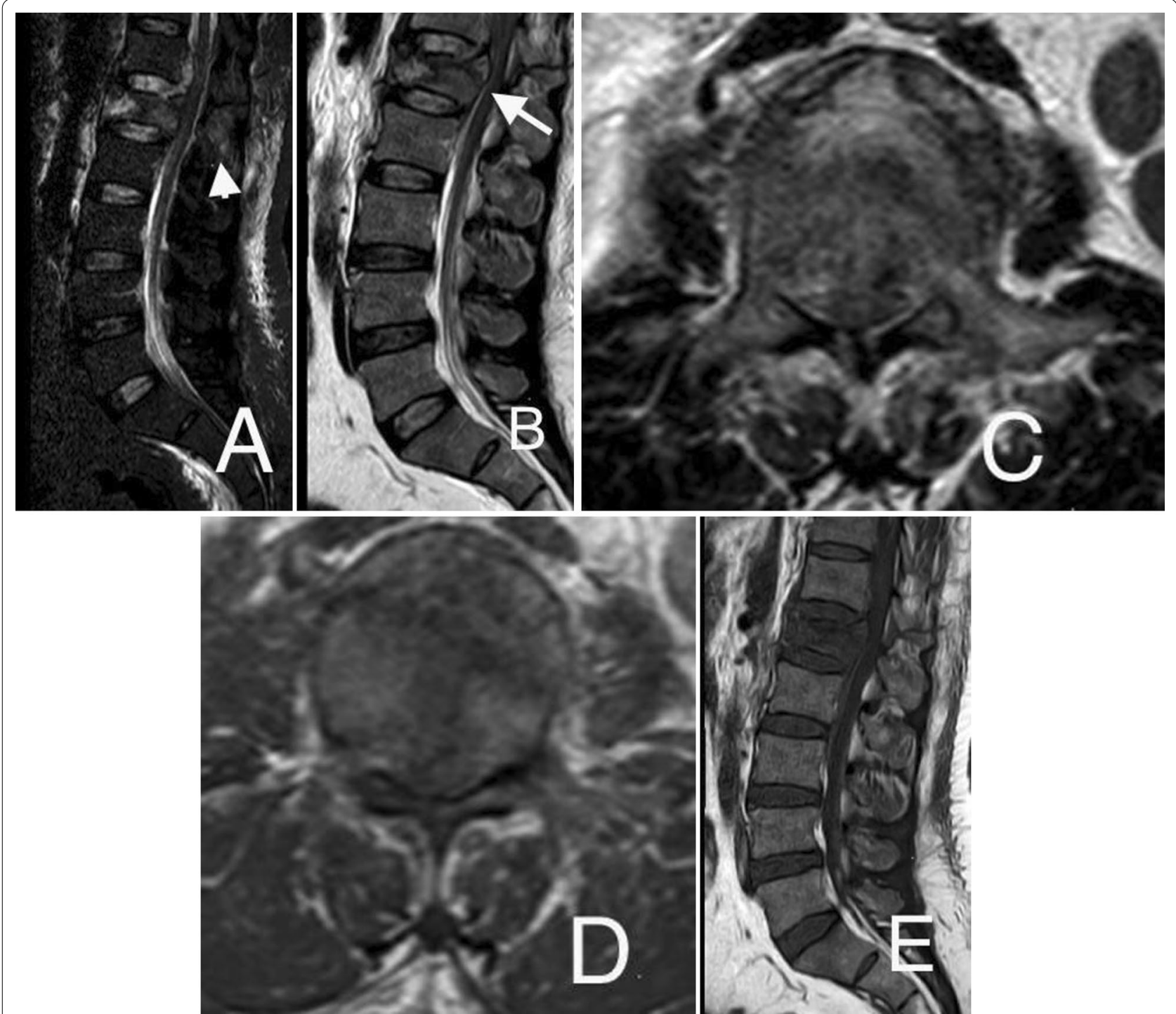

Fig. 6 A 48-year-old male presented after motorcar accident. a Sagittal STIR shows L1 burst fracture with suspected PLC injury eliciting high SI (arrowhead). b Sagittal T2WI shows complete burst fracture involving both end plates and posterior wall of vertebral body (comminuted) (white arrow). c Axial T2WI. d Axial T1WI, e Sagittal T1WI, TLICS score was 4, yet TL AOSIS=A4 N2, score was 7, this case shows the advantage of TL AOSIS over TLICS in accurate morphology description

\section{Conclusion}

Both TL AOSIS and TLICS have very close results in their reliability for guiding treatment strategy, yet TL AOSIS matched treatment decision recommendation more than TLICS, with sensitivity more than TLICS, while TLICS has specificity more than TL AOSIS.

\section{Abbreviations}

TLICS: Thoracolumbar injury classification and severity score system; TL AOSIS: Thoracolumbar AO Spine injury score; PLC injury: Posterior ligamentous complex injury.

\section{Acknowledgements}

None.

\section{Authors' contributions}

MMSS: Reviewing literature, data collection and analysis, perform statistical analysis, write and revise the manuscript, prepare figure and tables. MAN: Reviewing literature, data analysis, share in statistical analysis, data analysis, manuscript editing. All authors read and approved the final manuscript.

\section{Funding}

All authors declare that no funding or any source of financial interest. 


\section{Availability of data and materials}

Available on request with the corresponding author. The authors declare that they had full access to all data in this study and the authors take complete responsibility for the integrity of the data and the accuracy of the data analysis.

\section{Declarations}

\section{Ethics approval and consent to participate}

Not applicable as this was a retrospective study.

\section{Consent for publication}

All patients included in this research were fully conscious and older than 18-year-old and gave written informed consent to publish the data contained within this study. All researchers are accepting to publish this original article. This study is not previously published.

\section{Competing interests}

The authors declare that they have no competing interests.

Received: 13 September 2021 Accepted: 6 February 2022

Published online: 11 February 2022

\section{References}

1. Wilson JR, Forgione N, Fehlings MG (2013) Emerging therapies for traumatic spinal cord injury. CMAJ 185(6):485-492. https://doi.org/10.1503/ cmaj.121206

2. Wood K, Buttermann G, Mehbod A, Garvey T, Jhanjee R, Sechriest V (2003) Operative compared with nonoperative treatment of a thoracolumbar burst fracture without neurological deficit. A prospective, randomized study. J Bone Joint Surg Am 85(5):773-781. https://doi.org/10.2106/ 00004623-200305000-00001

3. Wang $H$, Zhang Y, Xiang Q, Wang X, Li C, Xiong H, Zhou Y (2012) Epidemiology of traumatic spinal fractures: experience from medical universityaffiliated hospitals in Chongqing, China, 2001-2010. J Neurosurg Spine 17(5):459-468. https://doi.org/10.3171/2012.8.SPINE111003

4. Kepler CK, Vaccaro AR, Schroeder GD, Koerner JD, Vialle LR, Aarabi B, Rajasekaran S, Bellabarba C, Chapman JR, Kandziora F, Schnake KJ, Dvorak MF, Reinhold M, Oner FC (2016) The thoracolumbar AOSpine injury score. Glob Spine J 6(4):329-334. https://doi.org/10.1055/s-0035-1563610

5. Ryken TC, Hadley MN, Walters BC, Aarabi B, Dhall SS, Gelb DE, Hurlbert RJ, Rozzelle CJ, Theodore N (2013) Radiographic assessment. Neurosurgery 72(3):54-72. https://doi.org/10.1227/NEU.0b013e318276edee

6. Joaquim AF, Fernandes YB, Cavalcante RA, Fragoso RM, Honorato DC, Patel AA (2011) Evaluation of the thoracolumbar injury classification system in thoracic and lumbar spinal trauma. Spine (Phila Pa 1976) 36(1):33-36. https://doi.org/10.1097/BRS.0b013e3181c95047

7. Joaquim AF, Ghizoni E, Tedeschi H, Batista UC, Patel AA (2014) Clinical results of patients with thoracolumbar spine trauma treated according to the thoracolumbar injury classification and severity score. J Neurosurg 20(5):562-567. https://doi.org/10.3171/2014.2.SPINE121114

8. Vaccaro AR, Lehman RA, Hurlbert RJ, Anderson PA, Harris M, Hedlund R, Harrop J, Dvorak M, Wood K, Fehlings MG, Fisher C, Zeiller SC, Anderson DG, Bono CM, Stock GH, Brown AK, Kuklo T, Öner Fc (2005) A new classification of thoracolumbar injuries: the importance of injury morphology, the integrity of the posterior ligamentous complex, and neurologic status. Spine 30(20):2325-2333. https://doi.org/10.1097/01.brs.00001 82986.43345.cb

9. Van Middendorp JJ, Patel AA, Schuetz M, Joaquim AF (2013) The precision, accuracy and validity of detecting posterior ligamentous complex injuries of the thoracic and lumbar spine: a critical appraisal of the literature. Eur Spine J 22(3):461-474. https://doi.org/10.1007/ s00586-012-2602-7

10. Magerl F, Aebi M, Gertzbein SD, Harms J, Nazarian S (1994) A comprehensive classification of thoracic and lumbar injuries. Eur Spine J 3(4):184201. https://doi.org/10.1007/BF02221591
11. Vaccaro AR, Oner C, Kepler CK, Dvorak M, Schnake K, Bellabarba C, Reinhold M, Aarabi B, Kandziora F, Chapman J, Shanmuganathan R, Fehlings $M$, Vialle L (2013) AO spine thoracolumbar spine injury classification system: fracture description, neurological status, and key modifiers. Spine (Phila Pa 1976) 38(23):2028-2037. https://doi.org/10.1097/BRS.0b013 e3182a8a381

12. Vaccaro AR, Schroeder GD, Kepler CK, Cumhur Oner F, Vialle LR, Kandziora F, Koerner JD, Kurd MF, Reinhold M, Schnake KJ, Chapman J, Aarabi B, Fehlings MG, Dvorak MF (2016) The surgical algorithm for the AOSpine thoracolumbar spine injury classification system. Eur Spine J 25(4):10871094. https://doi.org/10.1007/s00586-015-3982-2

13. Pizones J, Sánchez-Mariscal F, Zúñiga L, Alvarez P, Izquierdo E (2013) Prospective analysis of magnetic resonance imaging accuracy in diagnosing traumatic injuries of the posterior ligamentous complex of the thoracolumbar spine. Spine (Phila Pa 1976) 38(9):745-751. https://doi. org/10.1097/BRS.0b013e31827934e4

14. Pneumaticos SG, Karampinas PK, Triantafilopoulos G, Koufos S, Polyzois V, Vlamis J (2016) Evaluation of TLICS for thoracolumbar fractures. Eur Spine J 25(4):1123-1127. https://doi.org/10.1007/s00586-015-3889-y

15. Yuksel MO, Gurbuz MS, Is M, Somay H (2018) Is the thoracolumbar injury classification and severity score (TLICS) superior to the AO thoracolumbar injury classification system for guiding the surgical management of unstable thoracolumbar burst fractures without neurological deficit? Turk Neurosurg 28(1):94-98. https://doi.org/10.5137/1019-5149.JTN. 19094-16.2

16. Dodwad S-NM, Dodwad S-JM, Wisneski R, Khan SN (2015) Retrospective analysis of thoracolumbar junction injuries using the thoracolumbar injury severity and classification score, American spinal injury association class, injury severity score, age, sex, and length of hospitalization. J Spinal Disord Tech 28:410-416. https://doi.org/10.1097/BSD.0b013e3182a14743

17. An Z, Zhu Y, Wang G, Wei H, Dong L (2020) Is the thoracolumbar AOSpine injury score superior to the thoracolumbar injury classification and severity score for guiding the treatment strategy of thoracolumbar spine injuries? World Neurosurg 137:e493-e498. https://doi.org/10.1016/j. wneu.2020.02.013 (Epub 2020 Feb 11 PMID: 32058120)

18. Schnake KJ (2014) Expert's comment concerning grand rounds case entitled "Progressive kyphotic deformity in comminuted burst fractures treated non-operatively: the Achilles tendon of the thoracolumbar injury classification and severity score (TLICS)" (T.A. Mattei, J. Hanov. European Spine Journal) 23(11):2263-2264. https://doi.org/10.1007/ s00586-014-3591-5

\section{Publisher's Note}

Springer Nature remains neutral with regard to jurisdictional claims in published maps and institutional affiliations.

\section{Submit your manuscript to a SpringerOpen ${ }^{\circ}$ journal and benefit from:}

- Convenient online submission

- Rigorous peer review

- Open access: articles freely available online

- High visibility within the field

- Retaining the copyright to your article

Submit your next manuscript at springeropen.com 